Annales Geophysicae, 23, 3229-3235, 2005

SRef-ID: 1432-0576/ag/2005-23-3229

(C) European Geosciences Union 2005

\title{
A complex autoregressive model and application to monthly temperature forecasts
}

\author{
X. Gu${ }^{1}$ and J. Jiang ${ }^{2}$ \\ ${ }^{1}$ State Key Laboratory of Sever Weather, Chinese Academy of Meteorological Sciences, Beijing, 100081, China \\ ${ }^{2}$ Training Center of China Meteorological Administration, Beijing, 100081, China
}

Received: 26 June 2005 - Revised: 19 September 2005 - Accepted: 20 September 2005 - Published: 30 November 2005

\begin{abstract}
A complex autoregressive model was established based on the mathematic derivation of the least squares for the complex number domain which is referred to as the complex least squares. The model is different from the conventional way that the real number and the imaginary number are separately calculated. An application of this new model shows a better forecast than forecasts from other conventional statistical models, in predicting monthly temperature anomalies in July at 160 meteorological stations in mainland China. The conventional statistical models include an autoregressive model, where the real number and the imaginary number are separately disposed, an autoregressive model in the real number domain, and a persistence-forecast model.
\end{abstract}

Keywords. Ionosphere (Modeling and forecasting) - General (Instruments useful in three or more fields)

\section{Introduction}

The classical least-squares method in the real number domain is widely employed in statistics for establishing regression models (e.g. Kendall and Stuart, 1976; Montgomery and Peck, 1982; Kern et al., 1987; Kleinbaum et al., 1988), even in the mathematics-physical method for calculating spherical harmonic coefficients (e.g. Ge et al., 1980). However, few reports of the complex least-squares method and the complex autoregressive model were found in the atmospheric or geophysical sciences before. As is well-known, once a statistical method has been extended to the complex number domain, it has gotten some new functions and gives more information. For example, the CEOF (Complex Empirical Orthogonal Function), which is an extension from the EOF (Empirical Orthogonal Function) in the real number domain

Correspondence to: $\mathrm{X} . \mathrm{Gu}$

(guxq@cams.cma.gov.cn) to the complex number domain, acquires a new function of decomposing variations of patterns and phase of the traveling waves on various space scales (e.g. Rasmusson et al., 1981; Barnett, 1983), while the EOF decomposes only the stationary waves in an element field in meteorology or geophysics. It should obtain an improvement when the classical least-squares method and the autoregressive model were extended to the complex number domain.

The spherical harmonic coefficient in the global spectrum model in meteorology is in the complex number. Gu (1998) derived the complex least-squares method to have resolved complex auto-memory coefficients, then established a global auto-memory spectrum model, and obtained a good forecasting effect for a 500-mb height field (Cao and $\mathrm{Gu}, 2001$ ).

In this article, we derive the complex least-squares method in more detail in Sect. 2. Then we develop a complex autoregressive model of predicting monthly temperature anomalies in Sect. 3. Section 4 applies the complex regressive model to temperature anomalies for July at 160 meteorological stations in mainland China, and compares it to three other conventional statistical forecast models, to show a practicable improvement. Concluding remarks are presented in Sect. 5.

\section{The complex least squares: mathematical derivation}

In general, let $y$ be a complex predict- and, $x_{1}, x_{2}, \ldots, x_{p}$ be complex predictors numbered $p$ in all. They are all in the complex anomalies. A complex multiple regression equation for the complex anomalies is written as

$y=\beta_{1} x_{1}+\beta_{2} x_{2}+\cdots+\beta_{p} x_{p}+e$,

where $\beta_{1}, \beta_{2}, \ldots \beta_{p}$ are complex coefficients, $e$ is a complex error term. The question to be solved is how to determine the estimates of $b_{1}, b_{2}, \ldots b_{p}$ of the complex coefficients $\beta_{1}, \beta_{2}$, $\ldots \beta_{p}$ 
In order to describe the relationship between $y$ and $x_{1}, x_{2}, \ldots, x_{p}$, i.e. to solve the complex coefficients $\beta_{1}, \beta_{2}$, $\ldots \beta_{p}$, we suppose that $n$ is the sampling number, that

$y_{1}, y_{2}, \ldots y_{n}$

are the elements of the sample of predictand $y$, and that

$x_{i 1}, x_{i 2}, \ldots x_{i p},(i=1,2, \ldots n)$

are the sample elements of the predictors corresponding to the predictand $y_{i}$ at the i-th sampling sequence. Under the assumption of linear regression Eq. (1), we have a set of equations:

$\left\{\begin{array}{c}y_{1}=\beta_{1} x_{11}+\beta_{2} x_{12}+\cdots+\beta_{p} x_{1 p}+e_{1} \\ y_{2}=\beta_{2} x_{21}+\beta_{2} x_{22}+\cdots+\beta_{p} x_{2 p}+e_{2} \\ \vdots \\ y_{n}=\beta_{1} x_{n 1}+\beta_{2} x_{n 2}+\cdots+\beta_{p} x_{n p}+e_{n}\end{array}\right.$

and in matrix form:

$\boldsymbol{Y}=\mathbf{X} \beta+\mathbf{e}$,

where $Y, \beta, e$ are vectors of complex variables:

$\boldsymbol{Y}=\left(\begin{array}{c}y_{1} \\ y_{2} \\ \vdots \\ y_{n}\end{array}\right), \quad \boldsymbol{\beta}=\left(\begin{array}{c}\beta_{1} \\ \beta_{2} \\ \vdots \\ \beta_{p}\end{array}\right), \quad \boldsymbol{e}=\left(\begin{array}{c}e_{1} \\ e_{2} \\ \vdots \\ e_{n}\end{array}\right)$

and $X$ is a complex matrix of the predictors:

$\mathbf{X}=\left(\begin{array}{cccc}x_{11} & x_{12} & \cdots & x_{1 p} \\ x_{21} & x_{22} & \cdots & x_{2 p} \\ \vdots & \vdots & \vdots & \vdots \\ x_{n 1} & x_{n 2} & \cdots & x_{n p}\end{array}\right)$

where every element $y_{i}, \beta_{j}, e_{i}, x_{i j},(i=1, \ldots, n, j=1, \ldots, p)$ is a complex number.

In order to resolve the estimates $b_{j}$ of $\beta_{j},(j=1, \ldots, p)$, let the square sum of all errors of the fitted value $\hat{y}_{i}$ in the mode from the actual value $y_{i}$, i.e.

$Q=\sum_{i=1}^{n}\left|y_{i}-\hat{y}_{i}\right|^{2}$

be minimized.

The $Q$ is a non-negatively quadratic term of the $b_{1}, b_{2}, \ldots$, $b_{p}$. According to the extremum principle in the differential, the minimum value exists. We may have

$$
\left\{\begin{array}{c}
\frac{\partial Q}{\partial b_{1}}=0 \\
\frac{\partial Q}{\partial b_{2}}=0 \\
\vdots \\
\frac{\partial Q}{\partial b_{p}}=0
\end{array} .\right.
$$

Table 1. The average of the ACC and the RMSE.

\begin{tabular}{lllll}
\hline & M 1 & M 2 & M 3 & M 4 \\
\hline ACC & 0.185 & 0.089 & 0.061 & 0.064 \\
RMSE(C) & 1.079 & 1.113 & 1.147 & 1.449 \\
\hline
\end{tabular}

In this way, the complex least-squares method determines the estimates $b_{1}, b_{2}, \ldots, b_{p}$ of the complex coefficients $\beta_{1}$, $\beta_{2}, \ldots \beta_{p}$. The $b_{1}, b_{2}, \ldots, b_{p}$ are all in the complex number. Equation (4) may be written in a matrix form:

$$
\begin{aligned}
\mathbf{Q} & =(\mathbf{Y}-\hat{\mathbf{Y}})^{\mathbf{T}} \overline{(\mathbf{Y}-\hat{\mathbf{Y}})} \\
& =(\mathbf{Y}-\mathbf{X B})^{\mathbf{T}} \overline{(\mathbf{Y}-\mathbf{X B})} \\
& =\left(\mathbf{Y}^{\mathbf{T}}-\mathbf{X}^{\mathbf{T}} \mathbf{B}^{\mathbf{T}}\right)(\overline{\mathbf{Y}}-\overline{\mathbf{X B}}),
\end{aligned}
$$

where

$$
\begin{aligned}
\boldsymbol{Y} & =\left(\begin{array}{c}
y_{1} \\
y_{2} \\
\vdots \\
y_{n}
\end{array}\right), \quad \hat{\boldsymbol{Y}}=\left(\begin{array}{c}
\hat{y}_{1} \\
\hat{y}_{2} \\
\vdots \\
\hat{y}_{n}
\end{array}\right), \quad \boldsymbol{B}=\left(\begin{array}{c}
b_{1} \\
b_{2} \\
\vdots \\
b_{p}
\end{array}\right), \\
\mathbf{X} & =\left(\begin{array}{cccc}
x_{11} & x_{12} & \cdots & x_{1 p} \\
x_{21} & x_{22} & \cdots & x_{2 p} \\
\vdots & \vdots & \vdots & \vdots \\
x_{n 1} & x_{n 2} & \cdots & x_{n p}
\end{array}\right)
\end{aligned}
$$

Here ( $)^{T}$ represents a transpose of matrix or vector, $\overline{(~)}$ denotes a conjugate of the complex number, then Eq. (5) may be written as

$\frac{\partial \mathbf{Q}}{\partial \mathbf{B}}=0$.

Let

$b_{k}=b_{k}^{x}+b_{k}^{y} I \quad(k=1,2, \cdots, p)$,

then $\bar{b}_{k}=b_{k}^{x}-b_{k}^{y} I$,

where $b_{k}^{x}$ is the real part, $b_{k}^{y}$ is the imaginary part, both $b_{k}^{x}$ and $b_{k}^{y}$ themselves are real variables, I is the unit of imaginary number and $\bar{I} \times I=-1$. Thus the partial derivative of $Q$ with respect to $b$ may be decomposed into that with respect to the real part $b_{k}^{x}$ and to the imaginary part $b_{k}^{y}$, respectively, i.e. in detail:

$$
\begin{aligned}
& Q=\sum_{i=1}^{n}\left[\left(y_{i}-\sum_{j=1}^{p} b_{j} x_{i j}\right)\left(\bar{y}_{i}-\sum_{j=1}^{p} \bar{b}_{j} \bar{x}_{i j}\right)\right] \\
& \sum_{i=1}^{n}\left[y_{i} \bar{y}_{i}-y_{i} \sum_{j=1}^{p} \bar{b}_{j} \bar{x}_{i j}-\bar{y}_{i} \sum_{j=1}^{p} b_{j} x_{i j}+\sum_{j=1}^{p} b_{j} x_{i j} \sum_{j=1}^{p} \bar{b}_{j} \bar{x}_{i j}\right] \\
& =\sum_{i=1}^{n}\left\{y_{i} \bar{y}_{i}-y_{i} \sum_{j=1}^{p}\left[\left(b_{j}^{x}-b_{j}^{y} I\right) \bar{x}_{i j}\right]-\bar{y}_{i} \sum_{j=1}^{p}\left[\left(b_{j}^{x}+b_{j}^{y} I\right) x_{i j}\right]\right. \\
& \left.+\sum_{j=1}^{p}\left[\left(b_{j}^{x}+b_{j}^{y} I\right) x_{i j}\right] \sum_{j=1}^{p}\left[\left(b_{j}^{x}-b_{j}^{y} I\right) \bar{x}_{i j}\right]\right\} .
\end{aligned}
$$


The partial derivative of $Q$ with respect to the real part $b_{k}^{x}$ is as:

$$
\begin{aligned}
& \frac{\partial Q}{\partial b_{k}^{x}}= \sum_{i=1}^{n}\left\{\left[-y_{i} \bar{x}_{i k}-\bar{y}_{i} x_{i k}\right]+x_{i k} \sum_{j=1}^{p}\left[\left(b_{j}^{x}-b_{j}^{y} I t\right) \bar{x}_{i j} t\right]+\bar{x}_{i k} \sum_{j=1}^{p}\left[\left(b_{j}^{x}+b_{j}^{y} I\right) x_{i j} t\right]\right\} \\
&= \sum_{i=1}^{n}\left\{\left[-y_{i} \bar{x}_{i k}-\bar{y}_{i} x_{i k}\right]+\sum_{j=1}^{p}\left[b_{j}^{x}\left(x_{i k} \bar{x}_{i j}+\bar{x}_{i k} x_{i j}\right)\right]+\sum_{j=1}^{p}\left[b_{j}^{y}\left(\bar{x}_{i k} x_{i j}-x_{i k} \bar{x}_{i j}\right)\right] I\right\}=0 \\
&(k=1,2, \ldots p) .
\end{aligned}
$$

Then

$\sum_{j=1}^{p}\left\{b_{j}^{x}\left[\sum_{i=1}^{n}\left(x_{i k} \bar{x}_{i j}+\bar{x}_{i k} x_{i j}\right)\right]\right\}+I \sum_{j=1}^{p}\left\{b_{j}^{y}\left[\sum_{i=1}^{n}\left(\bar{x}_{i k} x_{i j}-x_{i k} \bar{x}_{i j}\right)\right]\right\}=\sum_{i=1}^{n}\left\{y_{i} \bar{x}_{i k}+\bar{y}_{i} x_{i k}\right]$

$$
(k=1,2, \ldots p) \text {. }
$$

While the partial derivative of $Q$ with respect to the imaginary part $b_{k}^{y}$

$$
\begin{gathered}
\frac{\partial Q}{\partial b_{k}^{y}}=\sum_{i=1}^{n}\left\{\left[y_{i} \bar{x}_{i k}-\bar{y}_{i} x_{i k}\right] I+x_{i k} I \sum_{j=1}^{p}\left[\left(b_{j}^{x}-b_{j}^{y} I\right) \bar{x}_{i j}\right]-\bar{x}_{i k} I \sum_{j=1}^{p}\left[\left(b_{j}^{x}+b_{j}^{y} I\right) x_{i j}\right]\right\} \\
=\sum_{i=1}^{n}\left\{\left[y_{i} \bar{x}_{i k}-\bar{y}_{i} x_{i k}\right] I+I \sum_{j=1}^{p}\left[b_{j}^{x}\left(x_{i k} \bar{x}_{i j}-\bar{x}_{i k} x_{i j}\right)\right]-I \sum_{j=1}^{p}\left[b_{j}^{y}\left(\bar{x}_{i k} x_{i j}+x_{i k} \bar{x}_{i j}\right)\right] I\right\}=0 \\
(k=1,2, \ldots p) .
\end{gathered}
$$

Then

$$
\begin{gathered}
\sum_{j=1}^{p}\left\{b_{j}^{x}\left[\sum_{i=1}^{n}\left(x_{i k} \bar{x}_{i j}-\bar{x}_{i k} x_{i j}\right)\right]\right\}-I \sum_{j=1}^{p}\left\{b_{j}^{y}\left[\sum_{i=1}^{n}\left(\bar{x}_{i k} x_{i j}+x_{i k} \bar{x}_{i j}\right)\right]\right\}=\sum_{i=1}^{n}\left\{\left[-y_{i} \bar{x}_{i k}+\bar{y}_{i} x_{i k}\right]\right\} \\
(k=1,2, \ldots p) .
\end{gathered}
$$

Combining Eqs. (11) and (13), we obtain:

$\sum_{j=1}^{p}\left[\left(b_{j}^{x}+b_{j}^{y} I\right) \sum_{i=1}^{n}\left(\bar{x}_{i k} x_{i j}\right)\right]=\sum_{i=1}^{n}\left(y_{i} \bar{x}_{i k}\right) k=1,2, \ldots, p$

Substituting Eq. (8) into Eq. (14), we obtain

$$
\sum_{j=1}^{p}\left[b_{j} \sum_{i=1}^{n}\left(\bar{x}_{i k} x_{i j}\right)\right]=\sum_{i=1}^{n}\left(y_{i} \bar{x}_{i k}\right) k=1,2, \ldots, p
$$

and may be written as the matrix form

$\overline{\mathbf{X}}^{\mathbf{T}} \mathbf{X B}=\overline{\mathbf{X}}^{\mathbf{T}} \mathbf{Y}$

or

$\mathbf{B}=\left(\overline{\mathbf{X}}^{\mathbf{T}} \mathbf{X}\right)^{-\mathbf{1}} \overline{\mathbf{X}}^{\mathbf{T}} \mathbf{Y}$,

where ()$^{-1}$ indicates a matrix inversion,

$\mathbf{X}=\left(\begin{array}{cccc}x_{11} & x_{12} & \cdots & x_{1 p} \\ x_{21} & x_{22} & \cdots & x_{2 p} \\ \vdots & \vdots & \vdots & \vdots \\ x_{n 1} & x_{n 2} & \cdots & x_{n p}\end{array}\right), \quad \boldsymbol{B}=\left(\begin{array}{c}b_{1} \\ b_{2} \\ \vdots \\ b_{p}\end{array}\right), \quad \boldsymbol{Y}=\left(\begin{array}{c}y_{1} \\ y_{2} \\ \vdots \\ y_{n}\end{array}\right)$

The elements $x_{i j}, b_{j}, y_{i}(i=1, \ldots, n, j=1, \ldots, p)$ are all in the complex number.
Note that Eq. (17) has acquired a conjugated calculation, which is the only difference from the classical least-squares method in the real number domain, as in Morrison (1983):

$\mathbf{B}=\left(\mathbf{X}^{\mathbf{T}} \mathbf{X}\right)^{-\mathbf{1}} \mathbf{X}^{\mathbf{T}} \mathbf{Y}$

\section{The complex autoregressive model}

Some scientists were used to calculating the real and imaginary number parts separately in the complex statistics (e.g. Hasselmann and Barnett, 1981; Jiang, 1983). However, we found that this way is not perfect, not only in the mathematical theory, but also in practice. In order to show the difference between the above-derived complex least squares and the method involving separate calculations, we apply the complex autoregressive model to forecasts of monthly temperature anomalies in July for 160 stations on mainland China, and compare that to three other conventional statistical models based on the same observational data during the period from 1951 to 2004.

For convenience of mathematical presentation in the complex number, and considering that the weather processes 


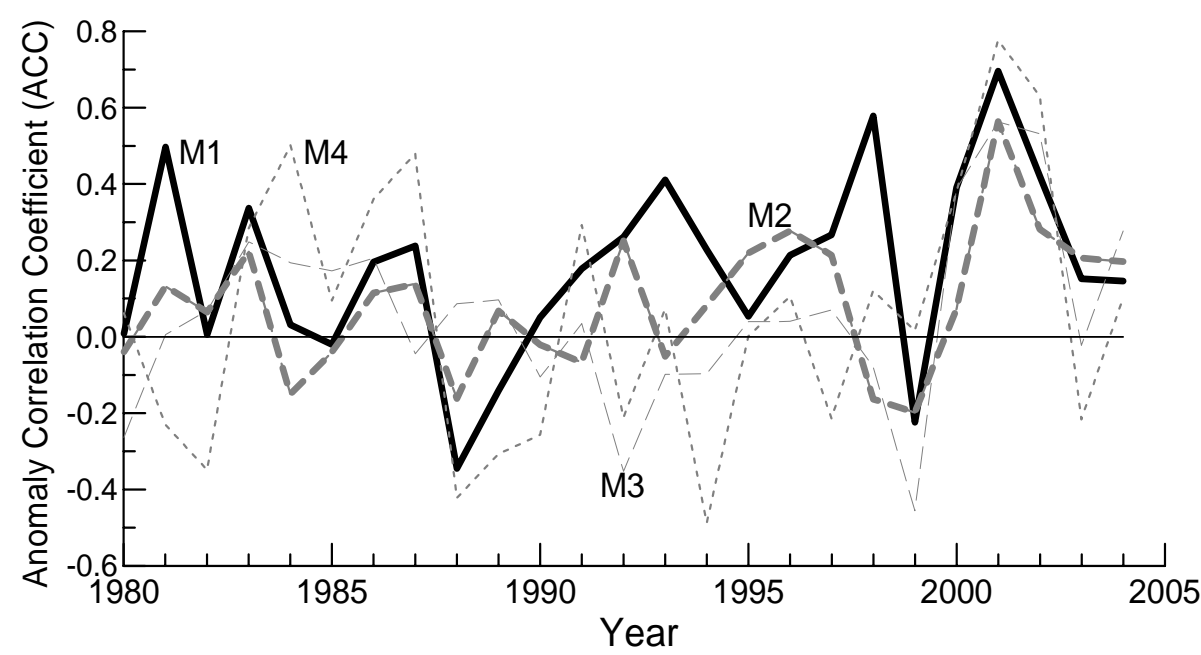

Fig. 1. Comparison of yearly ACC forecasted by the 4 models (M1: thick solid, M2: thick dashes, M3: thin dashes, M4: thin dots).

move usually in the direction from the northwest to southeast, or opposite as a typhoon in China, we put the 160 stations into one dimension sequence in order of latitude, then transformed the monthly temperature anomalies at 160 stations into a Fourier series (e.g. Li et al., 1982; Passi and Schumann, 1984; Xu, 1996) for each month to fit the spatial pattern:

$$
\begin{aligned}
T_{j, l} & =\sum_{k=1}^{N}\left[c_{k, l} \exp \left(I \frac{2 \pi}{N} j\right)\right], \\
c_{k, l} & =\sum_{k=1}^{N}\left[T_{j, l} \exp \left(-I \frac{2 \pi}{N} j\right)\right],
\end{aligned}
$$

where $T_{j, l}$ indicates the monthly temperature anomaly $\left({ }^{\circ} \mathrm{C}\right)$ at station $j$ and in year $l, j=1,2, \ldots, N=160$ represents the station number in order of latitude, and $l=1,2, \ldots, L=54$ is the time order of year. The $\exp \left(I \frac{2 \pi}{N} j\right)=\cos \left(\frac{2 \pi}{N} j\right)+I \sin \left(\frac{2 \pi}{N} j\right)$, and $I=\sqrt{-1}$ is the imaginary unit, $c_{k, l}$ is the Fourier coefficient:

$c_{k, l}=a_{k, l}+b_{k, l} I, \quad(k=1,2, \ldots, N)$.

Thus, the monthly temperature anomaly $T_{j, l}$ is fitted with the wave number $k$ to correspond to the spatial scale, and the $l$ corresponds to the year in the time series. Using historic data, we may obtain $c_{k, l}(k=1,2, \ldots, N)$ for a given year $l$ firstly, and construct a complex time series $c_{k, l}$ in the time order $l=1,2, \ldots, L=54$ for each wave number $k$ secondly. Then we may set up a complex autoregressive forecast model for the time series $c_{k, l}(l=1,2, \ldots, L=54)$ at every wave number $k$ :

$c_{k, l+1}=B_{0}+\sum_{j=1}^{p} B_{j} c_{k, l-j+1}$.

After determining the complex regressive coefficients $B_{j}(j=0,1, \ldots p)$ via Eq. (17), we may predict the Fourier coefficients for the next year, $c_{k, l+1}=a_{k, l+1}+b_{k, l+1} I$, via Eq. (22), and reconstruct the monthly temperature anomalies at 160 stations for the next year $T_{j, l+1}$ via Eq. (19). The prediction experiments suggest the autoregressive-order criterion $p=3$ (Akaike, 1969) in this work.

\section{Applications and comparison}

The historic monthly mean temperature anomalies in July $\left(T_{j, l}\right)$ were used to compute the Fourier coefficients $c_{k, l}$, following Eq. (20). All $c_{k, l}$ in the years before the year to be forecasted were taken as the training sample, to obtain the complex regression coefficients $B_{j}(j=0,1,2,3)$ via Eq. (17) for each wave number $k$. Yearly forecasting experiments were then carried out independently for 1980-2004 via Eq. (22), and the forecasted monthly mean temperature anomalies $\left(F_{j, l}\right)$ at 160 stations were finally reconstructed via Eq. (19).

The forecasted results were inspected with the anomaly correlation coefficient (ACC) between the predicted monthly mean temperature anomalies and the corresponding observations, and tested with the root-mean-square error (RMSE), too:

$$
\begin{gathered}
A C C=\frac{\sum_{j=1}^{N}\left[\left(F_{j}-M_{f c}\right)\left(T_{j}-M_{t c}\right)\right]}{\sqrt{\sum_{j=1}^{N}\left(F_{j}-M_{f c}\right)^{2} \sum_{i=1}^{N}\left(T_{j}-M_{t c}\right)^{2}}}, \\
R M S E=\sqrt{\frac{1}{N} \sum_{j=1}^{N}\left(F_{j}-T_{j}\right)^{2}},
\end{gathered}
$$




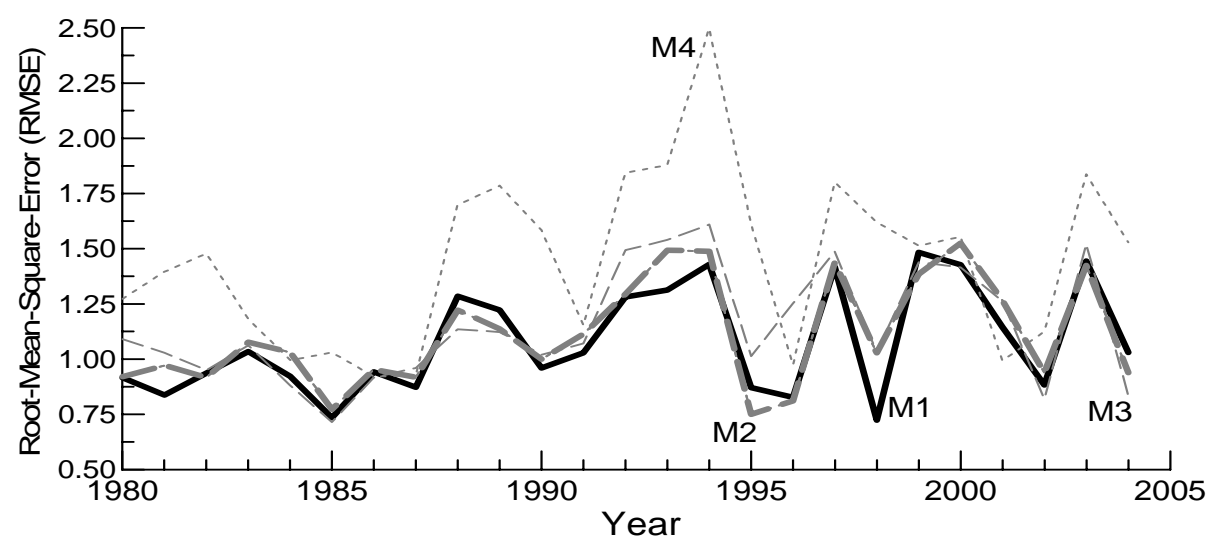

Fig. 2. As same as in Fig. 1 but for RMSE.

where $M_{f c}=\frac{1}{N} \sum_{j=1}^{N} F_{j}, M_{t c}=\frac{1}{N} \sum_{j=1}^{N} T_{j}$, and $F_{j}$ denotes the forecasted value for the station $j, T_{j}$ is the observation of the monthly temperature.

Table 1 compares averages of the ACC and of the RMSE over 1980-2004 among the 4 models, based on the same training data. The M1 (model 1) indicates the complex autoregressive model via Eq. (17). The M2 (model 2) denotes an autoregressive model (also $p=3$ ) where the real part and the imaginary part in the $c_{k, l}$ were computed separately via Eq. (18). The M3 (model 3) is a simple autoregressive model $(p=3)$ in the real number part only at each station via Eq. (18). The M4 (model 4) is a persistence forecast at each station. It shows that the complex autoregressive model (M1) produced $\mathrm{ACC}=0.185$ and $\mathrm{RMSE}=1.079 \mathrm{~mm}$ on average, which is obviously better than the three other models. The simple autoregressive model at each station (M3) is the worst one among the 4 models, on average, for the ACC, while the persistence forecast model (M4) is the worst one among the 4 models, on average, for the RMSE. As mentioned in Sect. 2, the M2, separately calculating the real and imaginary parts to obtain the regression coefficients is worse than the complex autoregressive model (M1) based on the complex least-square method Eq.(17) though it is somewhat better than the 2 other models, on average, for the ACC and RMSE. This suggests that separately calculating the real and imaginary parts to obtain a complex least square and a complex regression coefficient is not a perfect way.

Comparisons of the ACC for each year are plotted in Fig. 1. It shows that the complex autoregressive model (M1) given better predictions than the other models. The M1 gained the best forecast among the 4 models in 9 years, 1981, 1983, 1990, 1992-1994, 1997, 1998 and 2000, comparing to the three other models obtained in 2, 4 and 7 years, respectively. Also, the number of years with ACC $>0.40$ predicted by the M1 were 5 in 1981, 1993, 1998, 2000 and 2002, comparing to that by the other models of 1,2 and 4 years separately. While M1 failed to acchieve $\mathrm{ACC}<0$ in only 3 years, 1985, 1988 and 1989, comparing with the other models in
7 or 8 years. In addition, 2001 was predicted well by all 4 models with only a small difference.

Figure 2 illustrates yearly RMSE forecasted by the 4 models. The complex autoregressive model (M1) granted the smallest RMSE among the 4 models in 11 years, which is more than the other models: the M2 in 8 years, the M3 in 5 years and the M4 in 1 year, respectively. While the M1 predicted the largest RMSE among the 25-year forecasts at 1.484 in 1999, comparing to the M2 forecasted that at 1.525 in 2000, the M3 at 1.610 in 1994, and the M4 did that at 2.498 in 1994, separately. This suggests that the complex autoregressive model (M1) gave better forecasts of the smaller and more stable RMSE than the three other models, i.e. the M1 is the best one among the 4 models. On the other hand, the persistence forecast (M4) is apparently the worst one among the 4 models in the RMSE.

As an example, Fig. 3 illustrates maps of the monthly temperature anomaly patterns in July 1998. The upper panel presents the forecast from the complex autoregressive model (M1), while the lower panel is the corresponding observations. It shows agreements between the forecast and observations: (i) positive anomalies in most areas in mainland China with errors of intensities; (ii) higher positive anomalies in the northern end of Northeastern China, in the area between $115-120^{\circ} \mathrm{E}$ and $32-37^{\circ} \mathrm{N}$, around locations at $\left(117^{\circ} \mathrm{E}\right.$, $\left.26^{\circ} \mathrm{N}\right),\left(112^{\circ} \mathrm{E}, 27^{\circ} \mathrm{N}\right)$ and $\left(107^{\circ} \mathrm{E}, 41^{\circ} \mathrm{N}\right)$; (iii) lower positive anomalies in the areas between $100-110^{\circ} \mathrm{E}$ and 23 $26^{\circ} \mathrm{N}$, between $86-93^{\circ} \mathrm{E}$ and $28-32^{\circ} \mathrm{N}$, between $80-97^{\circ} \mathrm{E}$ and $40-49^{\circ} \mathrm{N}$ in China, around locations at $\left(118^{\circ} \mathrm{E}, 29^{\circ} \mathrm{N}\right)$ and $\left(107^{\circ} \mathrm{E}, 31^{\circ} \mathrm{N}\right)$; (iv) negative anomalies in the area of $87-88^{\circ} \mathrm{E}$ and $42-45^{\circ} \mathrm{N}$, though there are wrong forecasts in smaller areas between $88-101^{\circ} \mathrm{E}$ and $35-42^{\circ} \mathrm{N}$, around locations at $\left(105^{\circ} \mathrm{E}, 38^{\circ} \mathrm{N}\right),\left(77^{\circ} \mathrm{E}, 39^{\circ} \mathrm{N}\right),\left(129^{\circ} \mathrm{E}, 42^{\circ} \mathrm{N}\right)$ and $\left(117^{\circ} \mathrm{E}, 44^{\circ} \mathrm{N}\right)$.

The above comparisons show that the complex leastsquares method and the complex autoregressive model (M1), which is introduced in this paper, might be more reasonable and effective than the three conventional statistical models. This complex statistic model may be applied well to some 

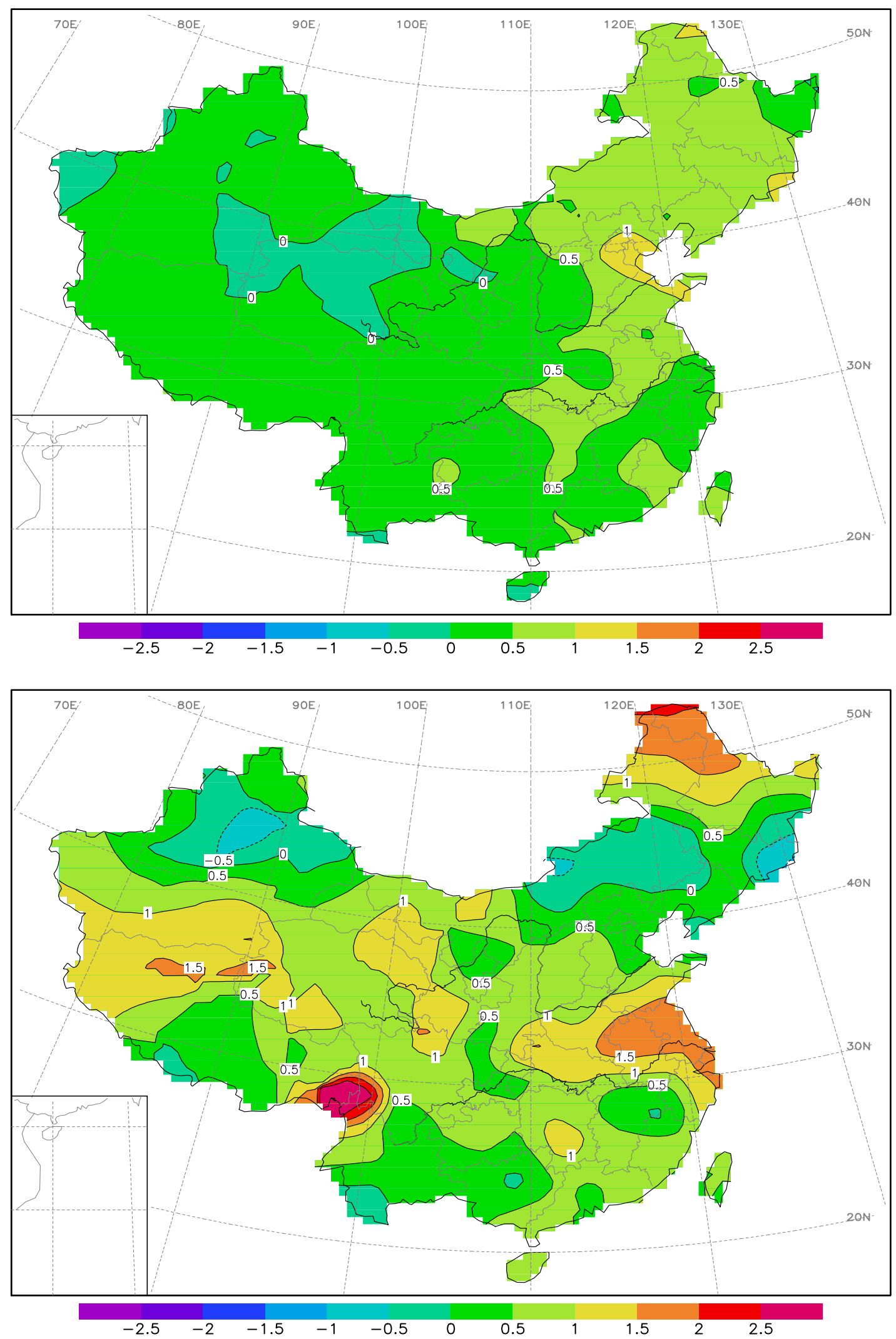

Fig. 3. Monthly temperature anomalies in July 1998 forecasted by the M1 (upper) and observations (lower). 
complex series, such as the Fourier transform and the spherical harmonic function.

In addition, there should be some improvements in the spatial pattern presentation, such as using the cluster analysis or the EOF/CEOF, instead of arranging the 160 stations in order of latitudes, to obtain a better result. For instance, we did forecast experiments with the M1 in a different spatial sequence order of stations, and yielded the average $\mathrm{ACC}=0.178$ in order of the original sequence number of the 160 stations, and the average $\mathrm{ACC}=0.174$ in order of longitude separately. This respect is an open question to be investigated in the future.

\section{Concluding remarks}

In this article, the complex least-squares method was mathematically derived and an autoregressive model of forecasting monthly temperature anomalies was established. The application in this work shows that using a complex number to fit a meteorological element field and predicting with the complex autoregressive model is effective in improving the forecast results.

Theoretical and applied results in this study suggest that separately calculating the real and the imaginary number parts to obtain a complex least square and a complex regression coefficients is a defective method.

The complex least-squares method extends the classical least-squares method from the real number domain into the complex number domain. It plays a key role and is an effective method to establish complex statistic models for dealing with complex series, such as the Fourier transform and the spherical harmonic function. Other similar statistical models, such as multiple regression and nonlinear regression, may be extended from the real number domain, into complex number domain, based on the complex least-squares method. This technology perhaps may be employed to other similar element fields in the geophysical sciences.

Acknowledgements. This work is supported by the National Natural Sciences Foundation of China, \#40175027, and by the Chinese National Key Program for Developing Basic Sciences, G1998040910. The authors would like to thank the referees for their ongoing support in improving this paper.

Topical Editor F. D'Andréa thanks two referees for their help in evaluating this paper.

\section{References}

Akaike, H.: Fitting autoregressive models for prediction, Annals Inst. Statist. Math., 27: 243-247, 1969.

Barnett, T. P.: Interaction of the monsoon and Pacific trade wind systems at interannual time scales, Part I: The equatorial Zone, Mom. Wea. Rev., 111, 756-773, 1983.

Cao, H. and Gu, X.: An integration forecast technology - Optimal harmonic method, Monthly Meteorology, 125(5), 3-6, 1999.

Cao, H. and $\mathrm{Gu}, \mathrm{X}$.: An improvement upon the medium-range Atmospheric circulation forecast with the self-memory spectral model(in Chinese), Advance in Nature Sciences, 11(3), 309-313, 2001.

Dover, T. G. J.: On the application of some stochastic models to precipitation forecasting, Quart. J. Roy.Met.Soc., 103, 177-189, 1977.

Ge, B., Shi, J., and Chou, J.: A Dynamical-statistical Long-term Weather Prediction Model Using Multi-time Historical Data, in: Preceedings of the 2nd National Conference on the Numerical Weather Prediction (in Chinese), Scieces Press, Beijing, 115$126,1980$.

Gu, X.: A spectral model based on atmospheric self-memorization principle, Chinese Science Bulletin, 43(20), 1692-1702, 1998.

Hasselmann, K. and Barnett, T. P.: Techniques of linear prediction for system with periodic statistics, J. Atmos. Sci., 38, 22752283, 1981.

Jiang, J.: The zonal harmonic of pentad mean $500 \mathrm{mb}$ height counters over the Northern Hemisphere and their prediction (in Chinese), Acta Meteorologica Sinica, 41(4), 433-443, 1983.

Kendall, M. G. and Stuart, M.: The Advanced Theory of Statistics I-III, Charles Griffin, London, 38-96, 1958-1976.

Kern, A. and Kern, M.: Handbook of Mathematics (in Chinese), translated by: Zhou, Q., Workers Press, Beijing, 530-534, 1987.

Kleinbaum, D. G., Kupper, L. L., and Muller, K. E.: Applied Regression Analysis and Other Multivaviable Methods, PWSKENT publishing company, Boston, 36-162, 1988.

Li, Q., Wang, N., Yi, D.: Numerical Analysis (in Chinese), Medium China Institute of Technology Press, Wuhan, 105-107, 1982.

Montgomery, D. C. and Peck, E. A.: Introduction to Linear Regression Analysis, John Wiley \& Sons, London, 43-85, 1982.

Morrison, D. F.: Applied Linear Statistical Methods, Prentice-Hall, Inc., Englewood Cliffs, New Jersey 07632, 228-254, 1983.

Passi, R. M. and Schumann, A. P.: Autoregressive modeling of quasi-biennial oscillation, preprints of 7th Conf. on probability and statistics in Atmos. Sci., Amer. Met. Soc., 176-181, 1984.

Rasmusson, E. M., Arkin, P. A., and Chen, W. Y.: Biennial Variation in syrface temperature over the United States as revealed by singular decomposition, Mom. Wea. Rev. 109, 587-598, 1981.

Sheng, J., Xie, S., and Pan, C.: Probability Theory and Mathematicphysic Statistics (2nd version, in Chinese), Advance Education Press, Beijing, 279-290, 1995.

$\mathrm{Xu}, \mathrm{S}$.: Collection of Frequently used Algorithm programs in the C Language (2nd edition), Qinghua University Press, Beijing, 266-270, 1996. 